\title{
The Hopf algebra of uniform block permutations
}

\author{
Marcelo Aguiar • Rosa C. Orellana
}

Received: 31 January 2007 / Accepted: 8 January 2008 / Published online: 24 January 2008

(C) Springer Science+Business Media, LLC 2008

\begin{abstract}
We introduce the Hopf algebra of uniform block permutations and show that it is self-dual, free, and cofree. These results are closely related to the fact that uniform block permutations form a factorizable inverse monoid. This Hopf algebra contains the Hopf algebra of permutations of Malvenuto and Reutenauer and the Hopf algebra of symmetric functions in non-commuting variables of Gebhard, Rosas, and Sagan. These two embeddings correspond to the factorization of a uniform block permutation as a product of an invertible element and an idempotent one.
\end{abstract}

Keywords Hopf algebra $\cdot$ Factorizable inverse monoid $\cdot$ Uniform block permutation $\cdot$ Set partition $\cdot$ Symmetric functions $\cdot$ Schur-Weyl duality

\section{Introduction}

A uniform block permutation of $[n]$ is a certain type of bijection between two set partitions of $[n]$. When the blocks of both partitions are singletons, a uniform block permutation is simply a permutation of $[n]$. Let $P_{n}$ be the set of uniform block permutations of $[n]$ and $S_{n}$ the subset of permutations of $[n]$. The set $P_{n}$ is a monoid in which the invertible elements are precisely the elements of $S_{n}$. These notions are reviewed in Section 2.

Aguiar supported in part by NSF grant DMS-0302423.

Orellana supported in part by the Wilson Foundation.

M. Aguiar ( $\varangle)$

Department of Mathematics, Texas A\&M University, College Station, TX 77843, USA

e-mail:maguiar@math.tamu.edu

R.C. Orellana

Department of Mathematics, Dartmouth College, Hanover, NH 03755, USA

e-mail: Rosa.C.Orellana@Dartmouth.EDU 
This paper introduces and studies a graded Hopf algebra based on the set of uniform block permutations of $[n]$ for all $n \geq 0$, by analogy with the graded Hopf algebra of permutations of Malvenuto and Reutenauer [21].

Let $V$ be a complex vector space. Classical Schur-Weyl duality states that the symmetric group algebra can be recovered from the diagonal action of $G L(V)$ on $V^{\otimes n}:$ if $\operatorname{dim} V \geq n$ then

$$
\mathbb{C} S_{n} \cong \operatorname{End}_{G L(V)}\left(V^{\otimes n}\right) .
$$

Malvenuto and Reutenauer deduce from here the existence of a multiplication among permutations as follows. Given $\sigma \in S_{p}$ and $\tau \in S_{q}$, view them as linear endomorphisms of the tensor algebra

$$
T(V):=\bigoplus_{n \geq 0} V^{\otimes n}
$$

by means of (1) ( $\sigma$ acts as 0 on $V^{\otimes n}$ if $n \neq p$, similarly for $\tau$ ). The tensor algebra is a Hopf algebra, so we can form the convolution product of any two linear endomorphisms:

$$
T(V) \stackrel{\Delta}{\rightarrow} T(V) \otimes T(V) \stackrel{\sigma \otimes \tau}{\longrightarrow} T(V) \otimes T(V) \stackrel{m}{\rightarrow} T(V),
$$

where $\Delta$ and $m$ are the coproduct and product of the tensor algebra. Since these two maps commute with the action of $G L(V)$, the convolution of $\sigma$ and $\tau$ belongs to $\operatorname{End}_{G L(V)}\left(V^{\otimes n}\right)$, where $n=p+q$. Therefore, there exists an element $\sigma * \tau \in \mathbb{C} S_{n}$ whose right action equals the convolution of $\sigma$ and $\tau$. This is the product of the algebra of permutations. It turns out that a suitable coproduct can also be defined. The result is the Hopf algebra of permutations of Malvenuto and Reutenauer.

The same argument can be applied to define a convolution product on the direct sum of the centralizer algebras $\operatorname{End}_{G}\left(V^{\otimes n}\right)$, starting from a linear action of a group $G$ on a vector space $V$. In this paper we consider one such instance in which in addition a compatible coproduct can also be defined.

Consider the complex reflection group $G(r, 1, m)$. The monomial representation is a certain linear action of this group on an $m$-dimensional space $V$ (Section 3.1). A result of Tanabe identifies the centralizer of $V^{\otimes n}$ with the monoid algebra of uniform block permutations (if $r$ and $m$ are big in comparison to $n$; see Proposition 3.1). The convolution product is therefore defined on the space $\bigoplus_{n} \mathbb{C} P_{n}$. An explicit description of this operation, similar to that for the convolution product of permutations, is given in Section 3.2, along with the definition of a compatible coproduct which turns this space into a graded Hopf algebra (Theorem 3.6). The Hopf algebra of permutations is a Hopf subalgebra (Proposition 3.7).

Except for the description of $\mathbb{C} P_{n}$ as a centralizer algebra, it is not necessary to work over the complex numbers. Accordingly, we work from the start over an arbitrary commutative ring $\mathbb{k}$. All modules considered will be free (having in fact a distinguished basis) and are therefore referred to as spaces.

The Hopf algebra structure of uniform block permutations is studied in Section 4.1. Proposition 4.1 states that this Hopf algebra is self-dual. This result is related to the fact that $P_{n}$ carries an involution which turns it into an inverse monoid. This 
is moreover a factorizable inverse monoid, which refers to the fact that any element is the product of an invertible element and an idempotent element. This is used in Section 4.2 to define a partial order on $P_{n}$ analogous to the weak order on $S_{n}$. Following [2], a second linear basis of the space $\mathbb{k} P_{n}$ is defined in Section 4.3 by performing Möbius inversion with respect to this partial order. The convolution product admits a simple description on this basis (Proposition 4.4) from which it follows that the algebra of uniform block permutations is free (and by self-duality also cofree) (Corollary 4.5).

It turns out that the Hopf algebra of uniform block permutations contains another distinguished Hopf subalgebra, namely the Hopf algebra of symmetric functions in non-commutative variables (Theorem 5.1). This algebra was introduced by Wolf [31]. It is based on the set of set partitions of $[n]$ for all $n \geq 0$. Sagan brought it to the forefront in a series of papers with Gebhard and Rosas [11, 12, 23]. Bergeron, Hohlweg, Rosas, and Zabrocki studied the Hopf algebra structure in [4, 5]. Connections between this and other combinatorial Hopf algebras are studied in [1]. The embedding of this Hopf algebra is in a sense complementary to the embedding of the algebra of permutations: permutations arise as the invertible elements of the monoid of uniform block permutations, while set partitions arise as the idempotent elements therein. This is discussed in Section 5.

This paper explores one aspect of the relationship between Schur-Weyl duality, diagram algebras, and inverse semigroups whose general study was started by Solomon [27] and continued by several authors [13-15, 19]. We thank the referees for bringing up these and other references to our attention.

\section{Uniform block permutations}

\subsection{Set partitions}

Let $n$ be a non-negative integer and let $[n]:=\{1,2, \ldots, n\}$. A set partition of $[n]$ is a collection of non-empty disjoint subsets of $[n]$, called blocks, whose union is $[n]$. For example, $\mathcal{A}=\{\{2,5,7\}\{1,3\}\{6,8\}\{4\}\}$, is a set partition of [8] with 4 blocks. We often specify a set partition by listing the blocks from left to right so that the sequence formed by the minima of the blocks is increasing, and by listing the elements within each block in increasing order. For instance, the set partition above will be denoted $\mathcal{A}=\{1,3\}\{2,5,7\}\{4\}\{6,8\}$. We use $\mathcal{A} \vdash[n]$ to indicate that $\mathcal{A}$ is a set partition of $[n]$.

The type of a set partition $\mathcal{A}$ of $[n]$ is the partition of $n$ formed by the sizes of the blocks of $\mathcal{A}$. The symmetric group $S_{n}$ acts on the set of set partitions of $[n]$ : given $\sigma \in S_{n}$ and $\mathcal{A} \vdash[n], \sigma(\mathcal{A})$ is the set partition whose blocks are $\sigma(A)$ for $A \in \mathcal{A}$. The orbit of $\mathcal{A}$ consists of those set partitions of the same type as $\mathcal{A}$. The stabilizer of $\mathcal{A}$ consists of those permutations that preserve the blocks, or that permute blocks of the same size. Therefore, the number of set partitions of type $1^{m_{1}} 2^{m_{2}} \ldots n^{m_{n}}\left(m_{i}\right.$ blocks of size $i$ ) is

$$
\frac{n !}{m_{1} ! \cdots m_{n} !(1 !)^{m_{1}} \cdots(n !)^{m_{n}}} .
$$



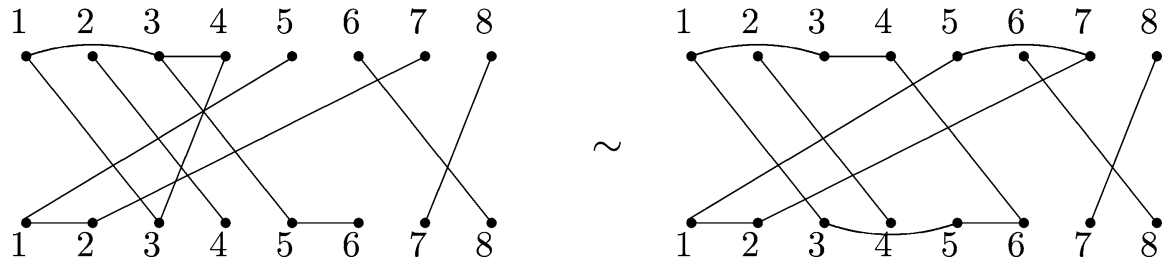

Fig. 1 Two graphs representing the same uniform block permutation

\subsection{The monoid of uniform block permutations}

The monoid (and the monoid algebra) of uniform block permutations has been studied by FitzGerald [10] and Kosuda [17, 18] in analogy to the partition algebra of Jones and Martin [16, 22].

A block permutation of $[n]$ consists of two set partitions $\mathcal{A}$ and $\mathcal{B}$ of $[n]$ with the same number of blocks and a bijection $f: \mathcal{A} \rightarrow \mathcal{B}$. For example, if $n=3$, $f(\{1,3\})=\{3\}$ and $f(\{2\})=\{1,2\}$ then $f$ is a block permutation. A block permutation is called uniform if it maps each block of $\mathcal{A}$ to a block of $\mathcal{B}$ of the same cardinality. For example, $f(\{1,3\})=\{1,2\}, f(\{2\})=\{3\}$ is uniform. Each permutation may be viewed as a uniform block permutation for which all blocks have cardinality 1 . In this paper we only consider block permutations that are uniform.

To specify a uniform block permutation $f: \mathcal{A} \rightarrow \mathcal{B}$ we must choose two set partitions $\mathcal{A}$ and $\mathcal{B}$ of the same type $1^{m_{1}} \ldots n^{m_{n}}$ and for each $i$ a bijection between the set of blocks of size $i$ of $\mathcal{A}$ and the set of blocks of size $i$ of $\mathcal{B}$. Since there are $m_{i}$ blocks of size $i$, it follows from (2) that the total number of uniform block permutations of $[n]$ is

$$
u_{n}:=\sum_{1^{m_{1}} \ldots n^{m_{n} \vdash n}}\left(\frac{n !}{(1 !)^{m_{1}} \cdots(n !)^{m_{n}}}\right)^{2} \frac{1}{m_{1} ! \cdots m_{n} !}
$$

where the sum runs over all partitions of $n$. Starting at $n=0$, the first values are

$$
1,1,3,16,131,1496,22482, \ldots
$$

This is sequence A023998 in [25]. These numbers and generalizations are studied in [24]; in particular, the following recursion is given in [24, equation (11)]:

$$
u_{n+1}=\sum_{k=0}^{n}\left(\begin{array}{l}
n \\
k
\end{array}\right)\left(\begin{array}{c}
n+1 \\
k
\end{array}\right) u_{k}, \quad u_{0}=1 .
$$

We represent uniform block permutations by means of graphs. For instance, either one of the two graphs in Figure 1 represents the uniform block permutation $f$ given by

$$
\{1,3,4\} \rightarrow\{3,5,6\},\{2\} \rightarrow\{4\},\{5,7\} \rightarrow\{1,2\},\{6\} \rightarrow\{8\}, \text { and }\{8\} \rightarrow\{7\} .
$$



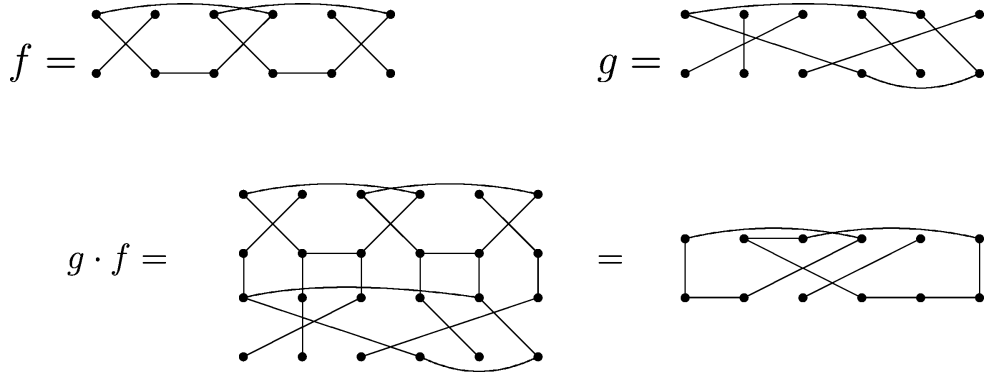

Fig. 2 Product of uniform block permutations

Different graphs may represent the same uniform block permutation. For a graph to represent a uniform block permutation $f: \mathcal{A} \rightarrow \mathcal{B}$ of $[n]$ the vertex set must consist of two copies of $[n]$ (top and bottom) and each connected component must contain the same number of vertices on the top as on the bottom. The set partition $\mathcal{A}$ is read off from the adjacencies on the top, $\mathcal{B}$ from those on the bottom, and $f$ from those in between.

The diagram of $f$ is the unique representing graph in which all connected components are cycles and the elements in each cycle are joined in order, as in the second graph of Figure 1.

The set $P_{n}$ of block permutations of $[n]$ is a monoid. The product $g \cdot f$ of two uniform block permutations $f$ and $g$ of $[n]$ is obtained by gluing the bottom of a graph representing $f$ to the top of a graph representing $g$. The resulting graph represents a uniform block permutation which does not depend on the graphs chosen. An example is given in Figure 2. Note that gluing the diagram of $f$ to the diagram of $g$ may not result in the diagram of $g \cdot f$.

The identity is the uniform block permutation that maps $\{i\}$ to $\{i\}$ for all $i$. Viewing permutations as uniform block permutations as above, we get that the symmetric group $S_{n}$ is a submonoid of $P_{n}$.

We recall a presentation of the monoid $P_{n}$ given in $[10,17,18]$. Consider the uniform block permutations $b_{i}$ and $s_{i}$ with diagrams
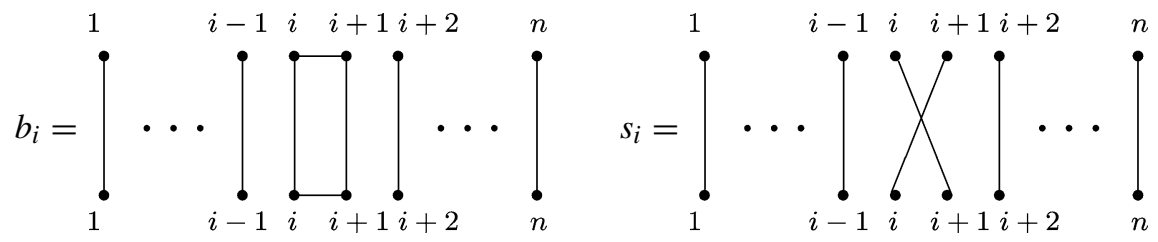

The monoid $P_{n}$ is generated by the elements $\left\{b_{i}, s_{i} \mid 1 \leq i \leq n-1\right\}$ subject to the following relations:

(1) $s_{i}^{2}=1, b_{i}^{2}=b_{i}, 1 \leq i \leq n-1$;

(2) $s_{i} s_{i+1} s_{i}=s_{i+1} s_{i} s_{i+1}, s_{i} b_{i+1} s_{i}=s_{i+1} b_{i} s_{i+1}, 1 \leq i \leq n-2$;

(3) $s_{i} s_{j}=s_{j} s_{i}, b_{i} s_{j}=s_{j} b_{i},|i-j|>1$;

(4) $b_{i} s_{i}=s_{i} b_{i}=b_{i}, 1 \leq i \leq n-1$;

(5) $b_{i} b_{j}=b_{j} b_{i}, 1 \leq i, j \leq n-1$. 
The submonoid generated by the elements $s_{i}, 1 \leq i \leq n-1$ is the symmetric group $S_{n}$, viewed as a submonoid of $P_{n}$ as above.

We will see in Sections 4.1 and 4.2 that $P_{n}$ is a factorizable inverse monoid. Therefore, a presentation for $P_{n}$ may also be derived from the results of [9].

\subsection{An ideal indexed by set partitions}

Let $\mathbb{k} P_{n}$ be the monoid algebra of $P_{n}$ over a commutative ring $\mathbb{k}$.

Given a set partition $\mathcal{A} \vdash[n]$, let $Z_{\mathcal{A}} \in \mathbb{k} P_{n}$ denote the sum of all uniform block permutations $f: \mathcal{A} \rightarrow \mathcal{B}$, where $\mathcal{B}$ varies:

$$
Z_{\mathcal{A}}:=\sum_{f: \mathcal{A} \rightarrow \mathcal{B}} f
$$

For instance,

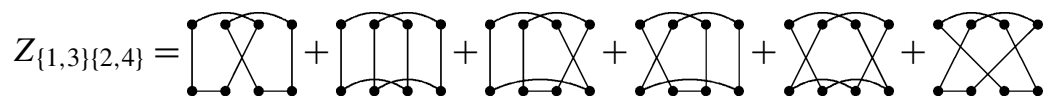

Given $\sigma \in S_{n}$ and $\mathcal{A} \vdash[n]$, the set partition $\sigma(\mathcal{A}) \vdash[n]$ was defined in Section 2.1.

Given $i \in[n]$, let $\mathcal{A}_{i}$ denote the set partition obtained by merging the blocks of $i$ and $i+1$ of $\mathcal{A}$ and keeping the other blocks of $\mathcal{A}$ unaltered.

Proposition 2.1 Let $\mathcal{A}$ be a set partition of $[n]$ and $\sigma$ a permutation of $[n]$. Then

$$
\sigma \cdot Z_{\mathcal{A}}=Z_{\mathcal{A}} \text { and } Z_{\mathcal{A}} \cdot \sigma=Z_{\sigma^{-1}(\mathcal{A})} \text {. }
$$

In addition,

$$
Z_{\mathcal{A}} \cdot b_{i}= \begin{cases}Z_{\mathcal{A}} & \text { if } i \text { and } i+1 \text { belong to the same block of } \mathcal{A} \\
\left(\begin{array}{c}
|A|+\left|A^{\prime}\right| \\
|A|
\end{array}\right) Z_{\mathcal{A}_{i}} & \text { if } i \text { and } i+1 \text { belong to different blocks } A \text { and } A^{\prime} \text { of } \mathcal{A} .\end{cases}
$$

Proof Let $f: \mathcal{A} \rightarrow \mathcal{B}$ be a summand of $Z_{\mathcal{A}}$. Then $\sigma \cdot f: \mathcal{A} \rightarrow \sigma(\mathcal{B})$. Thus left multiplication by $\sigma$ preserves the set of uniform block permutations with domain $\mathcal{A}$. Since $\sigma$ is invertible, this is a bijection. Therefore, $\sigma \cdot Z_{\mathcal{A}}=Z_{\mathcal{A}}$. Similarly, right multiplication by $\sigma$ is a bijection from the set of uniform block permutations with domain $\mathcal{A}$ to the set of uniform block permutations with domain $\sigma^{-1}(\mathcal{A})$. Hence $Z_{\mathcal{A}} \cdot \sigma=Z_{\sigma^{-1}(\mathcal{A})}$.

Let $A$ and $A^{\prime}$ be the blocks of $i$ and $i+1$ in the set partition $\mathcal{A}$ (they may coincide). Multiplying the uniform block permutation $f$ on the right by $b_{i}$ has the effect of connecting the vertices $i$ and $i+1$ in $\mathcal{A}$. The domain of $f \cdot b_{i}$ is therefore the set partition $\mathcal{A}_{i}$ and the codomain is the set partition $\overline{\mathcal{B}}$ obtained by merging the blocks $f(A)$ and $f\left(A^{\prime}\right)$ of $\mathcal{B}$. Moreover, $f \cdot b_{i}: \mathcal{A}_{i} \rightarrow \overline{\mathcal{B}}$ is the uniform block permutation such that $\left(f \cdot b_{i}\right)\left(A \cup A^{\prime}\right)=f(A) \cup f\left(A^{\prime}\right)$ and $\left(f \cdot b_{i}\right)\left(A^{\prime \prime}\right)=f\left(A^{\prime \prime}\right)$ on every other block $A^{\prime \prime}$ of $\mathcal{A}$.

On the other hand, let $g: \mathcal{A}_{i} \rightarrow \mathcal{C}$ be a uniform block permutation. The block $g\left(A \cup A^{\prime}\right)$ of $\mathcal{C}$ can be decomposed as the disjoint union of two subsets $C$ and $C^{\prime}$ 
with $|C|=|A|$ and $\left|C^{\prime}\right|=\left|A^{\prime}\right|$ in $\left(\begin{array}{c}\left|A+A^{\prime}\right| \\ |A|\end{array}\right)$ ways. For each such decomposition there is a unique set partition $\mathcal{B}$ and a unique uniform block permutation $f: \mathcal{A} \rightarrow \mathcal{B}$ such that $\overline{\mathcal{B}}=\mathcal{C}$ and $f \cdot b_{i}=g$. Therefore,

$$
Z_{\mathcal{A}} \cdot b_{i}=\left(\begin{array}{c}
\left|A+A^{\prime}\right| \\
|A|
\end{array}\right) Z_{\mathcal{A}_{i}} .
$$

Let $\mathcal{Z}_{n}$ denote the subspace of $\mathbb{k} P_{n}$ linearly spanned by the elements $Z_{\mathcal{A}}$ as $\mathcal{A}$ runs over all set partitions of $[n]$.

Corollary $2.2 \mathcal{Z}_{n}$ is a right ideal of the monoid algebra $\mathbb{k} P_{n}$.

\section{The Hopf algebra of uniform block permutations}

In this section we define the Hopf algebra of uniform block permutations. It contains the Hopf algebra of permutations of Malvenuto and Reutenauer as a Hopf subalgebra.

3.1 Schur-Weyl duality for uniform block permutations

Let $r$ and $m$ be positive integers. Let $C_{r}$ denote the cyclic group of order $r$ with generator $t$ :

$$
C_{r}:=\left\langle t \mid t^{r}=1\right\rangle \text {. }
$$

Consider the complex reflection group

$$
G(r, 1, m):=C_{r} 2 S_{m} .
$$

Let $V$ be the monomial representation of $G(r, 1, m)$. Thus, $V$ is an $m$-dimensional complex vector space with a basis $\left\{e_{1}, e_{2}, \ldots, e_{m}\right\}$ on which $G(r, 1, m)$ acts as follows:

$$
t \cdot e_{1}=e^{2 \pi i / r} e_{1}, \quad t \cdot e_{i}=e_{i} \text { for } i>1, \text { and } \sigma \cdot e_{i}=e_{\sigma(i)} \text { for } \sigma \in S_{m} .
$$

Consider now the diagonal action of $G(r, 1, m)$ on the tensor powers $V^{\otimes n}$,

$$
g \cdot\left(e_{i_{1}} e_{i_{2}} \cdots e_{i_{n}}\right)=\left(g \cdot e_{i_{1}}\right)\left(g \cdot e_{i_{2}}\right) \cdots\left(g \cdot e_{i_{n}}\right) .
$$

The centralizer of this representation has been calculated by Tanabe.

Proposition 3.1 [30] There is a right action of the monoid $P_{n}$ on $V^{\otimes n}$ determined by

$$
\left(e_{i_{1}} \cdots e_{i_{n}}\right) \cdot b_{j}=\delta\left(i_{j}, i_{j+1}\right) e_{i_{1}} \cdots e_{i_{n}} \text { and }\left(e_{i_{1}} \cdots e_{i_{n}}\right) \cdot \sigma=e_{i_{\sigma(1)}} \cdots e_{i_{\sigma(n)}}
$$

for $1 \leq i \leq n-1$ and $\sigma \in S_{n}$. This action commutes with the left action of $G(r, 1, m)$ on $V^{\bar{\otimes} n}$. Moreover, if $m \geq 2 n$ and $r>n$ then the resulting map

$$
\mathbb{C} P_{n} \rightarrow \operatorname{End}_{G(r, 1, m)}\left(V^{\otimes n}\right)
$$

is an isomorphism of algebras. 
Fig. 3 Concatenation of diagrams

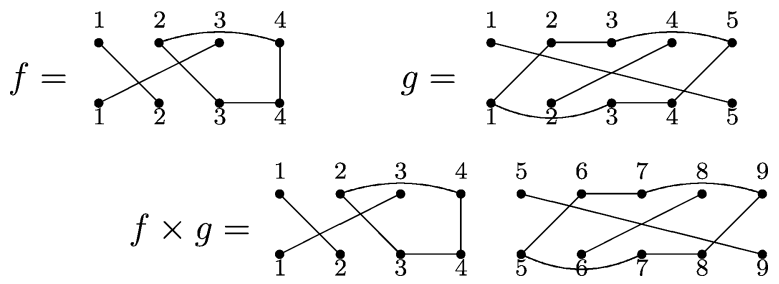

As explained in the introduction, this result can be used to deduce the existence of a product on the space

$$
\mathcal{P}:=\bigoplus_{n \geq 0} \mathbb{k} P_{n}
$$

for which the map

$$
\mathcal{P} \rightarrow \operatorname{End}(T(V))
$$

(resulting from (5)) is a morphism of algebras, where $\operatorname{End}(T(V))$ is viewed as an algebra under the convolution product. We proceed to describe the product on $\mathcal{P}$ in explicit terms and to enlarge this structure to a graded connected Hopf algebra.

\subsection{Product and coproduct of uniform block permutations}

Let $f$ and $g$ be uniform block permutations of $[p]$ and $[q]$ respectively. Adding $p$ to every entry in the diagram of $g$ and placing it to the right of the diagram of $f$ we obtain the diagram of a uniform block permutation of $[p+q]$, called the concatenation of $f$ and $g$ and denoted $f \times g$. Figure 3 shows an example.

Let $\operatorname{Sh}(p, q)$ denote the set of $(p, q)$-shuffles, that is, those permutations $\xi \in S_{p+q}$ such that

$$
\xi(1)<\xi(2)<\cdots<\xi(p) \text { and } \xi(p+1)<\xi(p+2)<\cdots<\xi(p+q) .
$$

Let $s h_{p, q} \in \mathbb{k} S_{p+q}$ denote the sum of all $(p, q)$-shuffles.

The product $*$ on $\mathcal{P}$ is defined by

$$
f * g:=s h_{p, q} \cdot(f \times g) \in \mathbb{k} P_{p+q}
$$

for all $f \in P_{p}$ and $g \in P_{q}$, and extended by linearity. It is easy to see that this product corresponds to convolution of endomorphisms of the tensor algebra via the map (5), when $\mathbb{k}=\mathbb{C}$.

For example,

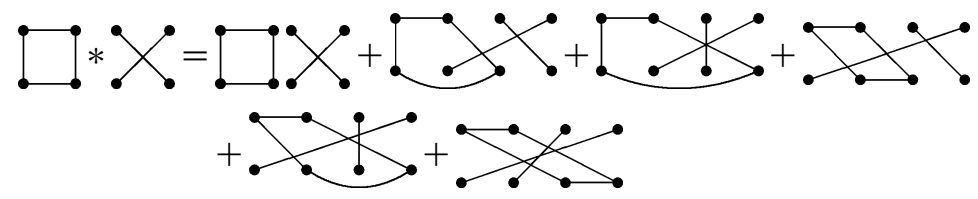

The set $P_{0}$ consists of the unique uniform block permutation of [0]. It is represented by the empty diagram, which we denote by $\emptyset$. It is the unit element for the product $*$. 
A breaking point of a set partition $\mathcal{B} \vdash[n]$ is an integer $i \in\{0,1, \ldots, n\}$ for which there exists a subset $S \subseteq \mathcal{B}$ such that

$$
\bigcup_{B \in S} B=\{1, \ldots, i\} \text { and (hence) } \bigcup_{B \in \mathcal{B} \backslash S} B=\{i+1, \ldots, n\} .
$$

Note that $i=0$ and $i=n$ are breaking points of any set partition $\mathcal{B}$.

Given a uniform block permutation $f: \mathcal{A} \rightarrow \mathcal{B}$, we say that $i$ is a breaking point of $f$ if it is a breaking point of $\mathcal{B}$, and we let $B(f)$ denote the set of breaking points of $f$. If $f$ is a permutation, that is if all blocks of $f$ are of size 1 , then $B(f)=\{0,1, \ldots, n\}$. In terms of the diagram of $f, i \in B(f)$ if it is possible to put a vertical line between the first $i$ and the last $n-i$ vertices in the bottom row without intersecting any edges joining bottom vertices.

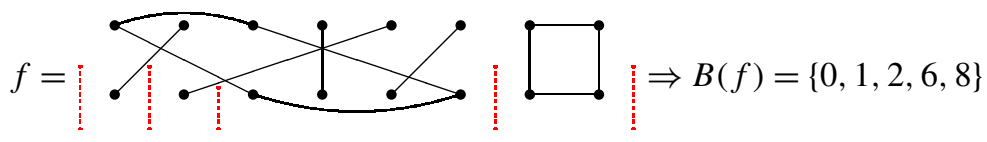

Lemma 3.2 If $i$ is a breaking point of $f$, then there exists a unique $(i, n-i)$-shuffle $\xi \in S_{n}$ and unique uniform block permutations $f_{(i)} \in P_{i}$ and $f_{(n-i)}^{\prime} \in P_{n-i}$ such that

$$
f=\left(f_{(i)} \times f_{(n-i)}^{\prime}\right) \cdot \xi^{-1} .
$$

Conversely, if such a decomposition exists, $i$ is a breaking point of $f$.

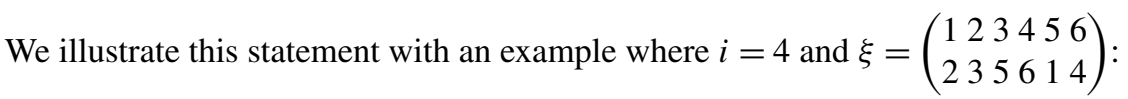

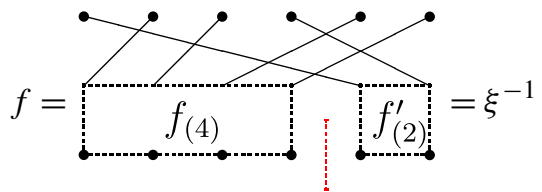

Proof Suppose $i$ is a breaking point of $f: \mathcal{A} \rightarrow \mathcal{B}$. Let $S$ be the subset of $\mathcal{B}$ as in (7). Write

$$
\bigcup_{B \in S} f^{-1}(B)=\left\{a_{1}, a_{2}, \ldots, a_{i}\right\} \quad \text { and } \bigcup_{B \in \mathcal{B} \backslash S} f^{-1}(B)=\left\{a_{i+1}, a_{i+2}, \ldots, a_{n}\right\}
$$

with $1 \leq a_{1}<a_{2}<\cdots<a_{i}, a_{i+1}<a_{i+2}<\cdots<a_{n} \leq n$. Define $\xi \in \operatorname{Sh}(i, n-i)$ by

$$
\xi(r)=a_{r} \quad \text { for } \quad r=1, \ldots, n .
$$

By construction, any element of $[i]$ is connected only to elements of $[i]$ in the diagram of $f \cdot \xi$. Therefore, there exist $f_{(i)} \in P_{i}$ and $f_{(n-i)}^{\prime} \in P_{n-i}$ such that $f \cdot \xi=f_{(i)} \times$ $f_{(n-i)}^{\prime}$. 
Assume that such a decomposition is given. Since $i$ is a breaking point of $f_{(i)} \times$ $f_{(n-i)}^{\prime}$ and the codomain of $f \cdot \xi$ is the same as the codomain of $f$, we have that $i$ is a breaking point of $f$. Hence we must have $\xi([i])=\left\{a_{1}, a_{2}, \ldots, a_{i}\right\}$, which makes the shuffle $\xi$ unique. Then $f_{(i)}$ and $f_{(n-1)}^{\prime}$ are determined from $f \cdot \xi=f_{(i)} \times f_{(n-i)}^{\prime}$.

We are now ready to define the coproduct on $\mathcal{P}$. Given $f \in P_{n}$ set

$$
\Delta(f):=\sum_{i \in B(f)} f_{(i)} \otimes f_{(n-i)}^{\prime},
$$

where $f_{(i)}$ and $f_{(n-i)}^{\prime}$ are as in Lemma 3.2. An example follows.

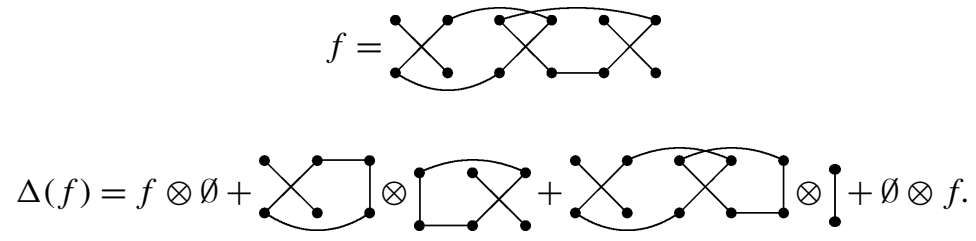

We define the counit $\varepsilon: \mathcal{P} \rightarrow \mathbb{k}$ by

$$
\varepsilon(f)= \begin{cases}1 & \text { if } f=\emptyset \in P_{0} \\ 0 & \text { if } f \in P_{n}, n \geq 1\end{cases}
$$

Remark 3.3 Recall that an element $x \in \mathcal{P}$ is called primitive if $\Delta(x)=x \otimes \emptyset+\emptyset \otimes$ $x$. Every uniform block permutation with breaking set $\{0, n\}$ is primitive, but there are other primitive elements in $\mathcal{P}$. For example, the following element of $\mathbb{k} P_{3}$ is primitive:

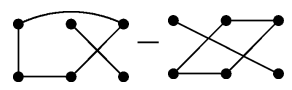

The primitive elements of $\mathcal{P}$ are determined in Section 4.3.

For the proof of the next theorem we need the following element of the symmetric group. Given $p, q \geq 0$, let $\xi_{p, q} \in S_{p+q}$ be the permutation

$$
\xi_{p, q}:=\left(\begin{array}{cccccccc}
1 & 2 & \ldots & p & p+1 & p+2 & \ldots & p+q \\
q+1 & q+2 & \ldots & q+p & 1 & 2 & \ldots & q
\end{array}\right) .
$$

This is a $(p, q)$-shuffle (it is in fact the maximum element of $\operatorname{Sh}(p, q)$ under the weak order; see Section 4.2). The diagram of $\xi_{3,4}$ is shown below.

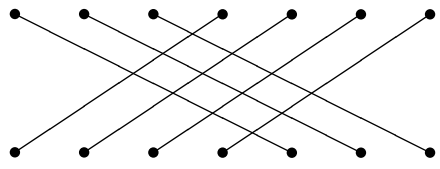

The inverse of $\xi_{p, q}$ is $\xi_{q, p}$. Let $1_{n} \in S_{n}$ denote the identity permutation. We need the following familiar properties. 
Lemma 3.4 Let $p, q \geq 0$. For any $f \in P_{q}, g \in P_{q}$, we have

$$
\xi_{p, q} \cdot(f \times g)=(g \times f) \cdot \xi_{p, q} .
$$

Lemma 3.5 Let $a, b, c, d \geq 0$. For any shuffles $\xi_{1} \in \operatorname{Sh}(a, b)$ and $\xi_{2} \in \operatorname{Sh}(c, d)$, the permutation

$$
\left(\xi_{1} \times \xi_{2}\right) \cdot\left(1_{a} \times \xi_{c, b} \times 1_{d}\right)
$$

is an $(a+c, b+d)$-shuffle. Conversely, let $p, q, r, s \geq 0$ be such that

$$
p+q=r+s
$$

Given a shuffle $\xi \in \operatorname{Sh}(p, q)$, there are unique numbers $a, b, c, d \geq 0$ and unique shuffles $\xi_{1} \in \operatorname{Sh}(a, b)$ and $\xi_{2} \in \operatorname{Sh}(c, d)$ such that

$$
a+b=r, \quad c+d=s, \quad a+c=p, \quad b+d=q,
$$

and

$$
\xi=\left(\xi_{1} \times \xi_{2}\right) \cdot\left(1_{a} \times \xi_{c, b} \times 1_{d}\right)
$$

Proof The first assertion is straightforward. For the converse, given $\xi \in \operatorname{Sh}(p, q)$, we have

$$
\xi^{-1}(\{1, \ldots, r\})=\{1, \ldots, a\} \cup\{p+1, \ldots, p+b\}
$$

for some $a, b \geq 0$ such that $a \leq p, b \leq q$, and $a+b=r$ (since $\xi$ is increasing on $\{1, \ldots, p\}$ and on $\{p+1, \ldots, p+q\})$. Let $c=p-a$ and $d=q-b$. A straightforward calculation shows that

$$
\xi \cdot\left(1_{a} \times \xi_{b, c} \times 1_{d}\right)=\xi_{1} \times \xi_{2}
$$

for some shuffles $\xi_{1} \in \operatorname{Sh}(a, b)$ and $\xi_{2} \in \operatorname{Sh}(c, d)$. This proves existence. The numbers $a$ and $b$ are uniquely determined from $\xi^{-1}(\{1, \ldots, r\})$ as above, and then $c, d$ and $\xi_{1}, \xi_{2}$ are determined as well.

Theorem 3.6 The graded vector space $\mathcal{P}$ equipped with the product $*$, coproduct $\Delta$, unit $\emptyset$, and counit $\varepsilon$, is a graded connected Hopf algebra.

Proof Associativity and coassociativity follow from basic properties of shuffles (for the product one may also appeal to (5) and associativity of the convolution product). The existence of the antipode is guaranteed in any graded connected bialgebra. The compatibility between $\Delta$ and $*$ requires a special argument.

Take $f \in \mathbb{k} P_{p}$ and $g \in \mathbb{k} P_{q}$. On the one hand we have

$$
\Delta(f * g) \stackrel{(6)}{=} \sum_{\xi \in \operatorname{Sh}(p, q)} \Delta(\xi \cdot(f \times g)) \stackrel{(8)}{=} \sum_{\substack{\xi \in \operatorname{Sh}(p, q) \\ r \in B(\xi \cdot(f \times g))}} \alpha \otimes \beta,
$$


here we have written, for each breaking point $r \in B(\xi \cdot(f \times g))$,

$$
\xi \cdot(f \times g) \cdot \eta=\alpha \times \beta,
$$

where $\alpha \in \mathbb{k} P_{r}, \beta \in \mathbb{k} P_{p+q-r}$, and $\eta \in \mathrm{Sh}(r, p+q-r)$ are the unique elements afforded by Lemma 3.2.

On the other hand, we have

$$
\begin{aligned}
\Delta(f) * \Delta(g) & =\sum_{\substack{a \in B(f) \\
b \in B(g)}}\left(f_{(a)}^{\prime} * g_{(b)}^{\prime}\right) \otimes\left(f_{(p-a)}^{\prime \prime} * g_{(q-b)}^{\prime \prime}\right) \\
& =\sum_{\substack{\xi_{1} \in \operatorname{Sh}(a, b) \\
\xi_{2} \in \operatorname{Sh}(p-a, q-b)}} \sum_{\substack{a \in B(f) \\
b \in B(g)}} \xi_{1} \cdot\left(f_{(a)}^{\prime} \times g_{(b)}^{\prime}\right) \otimes \xi_{2} \cdot\left(f_{(p-a)}^{\prime \prime} \times g_{(q-b)}^{\prime \prime}\right) .
\end{aligned}
$$

Here, for each pair of breaking points $a \in B(f)$ and $b \in B(g)$, we have written

$$
f \cdot \eta_{1}=f_{(a)}^{\prime} \times f_{(p-a)}^{\prime \prime} \quad \text { and } \quad g \cdot \eta_{2}=g_{(b)}^{\prime} \times g_{(q-b)}^{\prime \prime},
$$

as in Lemma 3.2, with $\eta_{1} \in \operatorname{Sh}(a, p-a)$ and $\eta_{2} \in \operatorname{Sh}(b, q-b)$.

We show that any summand in $\Delta(f) * \Delta(g)$ also occurs in $\Delta(f * g)$ and viceversa.

Given breaking points $a, b$ and shuffles $\eta_{1}, \eta_{2}$ as in (12), define

$$
\eta:=\left(\eta_{1} \times \eta_{2}\right) \cdot\left(1_{a} \times \xi_{b, p-a} \times 1_{q-b}\right) .
$$

In addition, given shuffles $\xi_{1} \in \operatorname{Sh}(a, b)$ and $\xi_{2} \in \operatorname{Sh}(p-a, q-b)$, define

$$
\xi:=\left(\xi_{1} \times \xi_{2}\right) \cdot\left(1_{a} \times \xi_{p-a, b} \times 1_{q-b}\right) .
$$

Then $\eta \in \operatorname{Sh}(a+b, p+q-a-b)$ and $\xi \in \operatorname{Sh}(p, q)$, by Lemma 3.5. Moreover, we have:

$$
\begin{aligned}
& \xi \cdot(f \times g) \cdot \eta=\left(\xi_{1} \times \xi_{2}\right) \cdot\left(1_{a} \times \xi_{p-a, b} \times 1_{q-b}\right) \cdot(f \times g) \\
& \cdot\left(\eta_{1} \times \eta_{2}\right) \cdot\left(1_{a} \times \xi_{b, p-a} \times 1_{q-b}\right) \\
&=\left(\xi_{1} \times \xi_{2}\right) \cdot\left(1_{a} \times \xi_{p-a, b} \times 1_{q-b}\right) \cdot\left(f \cdot \eta_{1}\right) \times\left(g \cdot \eta_{2}\right) \\
& \cdot\left(1_{a} \times \xi_{b, p-a} \times 1_{q-b}\right) \\
& \stackrel{(12)}{=}\left(\xi_{1} \times \xi_{2}\right) \cdot\left(1_{a} \times \xi_{p-a, b} \times 1_{q-b}\right) \cdot\left(f_{(a)}^{\prime} \times f_{(p-a)}^{\prime \prime} \times g_{(b)}^{\prime} \times g_{(q-b)}^{\prime \prime}\right) \cdot\left(1_{a} \times \xi_{b, p-a} \times 1_{q-b}\right) \\
& \stackrel{(10)}{=}\left(\xi_{1} \times \xi_{2}\right) \cdot\left(f_{(a)}^{\prime} \times g_{(b)}^{\prime} \times f_{(p-a)}^{\prime \prime} \times g_{(q-b)}^{\prime \prime}\right) \\
&=\left(\xi_{1} \times\left(f_{(a)}^{\prime} \times g_{(b)}^{\prime}\right)\right) \times\left(\xi_{2} \times\left(f_{(p-a)}^{\prime \prime} \times g_{(q-b)}^{\prime \prime}\right)\right) .
\end{aligned}
$$

Therefore, by Lemma 3.2, $a+b$ is a breaking point of $\xi \cdot(f \times g)$, and by (11),

$$
\xi_{1} \times\left(f_{(a)}^{\prime} \times g_{(b)}^{\prime}\right)=\alpha \quad \text { and } \quad \xi_{2} \times\left(f_{(p-a)}^{\prime \prime} \times g_{(q-b)}^{\prime \prime}\right)=\beta .
$$


This shows that every summand in $\Delta(f) * \Delta(g)$ also occurs in $\Delta(f * g)$.

Now assume that we are given a shuffle $\xi$ and a breaking point $r$ as in (11). We have to show that the summand $\alpha \otimes \beta$ of $\Delta(f * g)$ also appears in $\Delta(f) * \Delta(g)$. Let $s:=p+q-r$. Applying Lemma 3.5 we find numbers $a, b$ and shuffles $\xi_{1} \in \operatorname{Sh}(a, b)$ and $\xi_{2} \in \operatorname{Sh}(p-a, q-b)$ such that $a+b=r$ and

$$
\xi=\left(\xi_{1} \times \xi_{2}\right) \cdot\left(1_{a} \times \xi_{p-a, b} \times 1_{q-b}\right)
$$

We first claim that $a$ is a breaking point of $f$ and $b$ is a breaking point of $g$. Consider the diagram of $f$. Suppose there is an edge connecting one of the first $a$ bottom vertices to one of the last $p-a$ bottom vertices. Say the former is $i$ and the latter is $j$. Then in the diagram of $\xi \cdot(f \times g)$ there is an edge connecting the bottom vertices $\xi(i)$ and $\xi(j)$. But this contradicts the fact that $r$ is a breaking point of $\xi \cdot(f \times g)$, since from the proof of Lemma 3.5 we know that $\xi(i) \in\{1, \ldots, r\}$ and $\xi(j) \in\{r+1, \ldots, r+s\}$.

Thus $a \in B(f)$, and similarly $b \in B(g)$. Therefore, we can write

$$
f=\left(f_{(a)}^{\prime} \times f_{(p-a)}^{\prime \prime}\right) \cdot \eta_{1}^{-1} \quad \text { and } \quad g=\left(g_{(b)}^{\prime} \times g_{(q-b)}^{\prime \prime}\right) \cdot \eta_{2}^{-1}
$$

where the elements in the right hand side are afforded by Lemma 3.2. We calculate:

$$
\begin{aligned}
\xi \cdot(f \times g)= & \left(\xi_{1} \times \xi_{2}\right) \cdot\left(1_{a} \times \xi_{p-a, b} \times 1_{q-b}\right) \\
& \cdot\left(\left(f_{(a)}^{\prime} \times f_{(p-a)}^{\prime \prime}\right) \cdot \eta_{1}^{-1}\right) \times\left(\left(g_{(b)}^{\prime} \times g_{(q-b)}^{\prime \prime}\right) \cdot \eta_{2}^{-1}\right) \\
= & \left(\xi_{1} \times \xi_{2}\right) \cdot\left(1_{a} \times \xi_{p-a, b} \times 1_{q-b}\right) \\
& \cdot\left(f_{(a)}^{\prime} \times f_{(p-a)}^{\prime \prime} \times g_{(b)}^{\prime} \times g_{(q-b)}^{\prime \prime}\right) \cdot\left(\eta_{1}^{-1} \times \eta_{2}^{-1}\right) \\
\stackrel{(10)}{=}\left(\xi_{1} \times \xi_{2}\right) \cdot\left(f_{(a)}^{\prime} \times g_{(b)}^{\prime} \times f_{(p-a)}^{\prime \prime} \times g_{(q-b)}^{\prime \prime}\right) & \cdot\left(1_{a} \times \xi_{p-a, b} \times 1_{q-b}\right) \cdot\left(\eta_{1}^{-1} \times \eta_{2}^{-1}\right) \\
= & \left(\left(\xi_{1} \cdot\left(f_{(a)}^{\prime} \times g_{(b)}^{\prime}\right)\right) \times\left(\xi_{2} \cdot\left(f_{(p-a)}^{\prime \prime} \times g_{(q-b)}^{\prime \prime}\right)\right)\right) \\
& \cdot\left(1_{a} \times \xi_{p-a, b} \times 1_{q-b}\right) \cdot\left(\eta_{1}^{-1} \times \eta_{2}^{-1}\right) .
\end{aligned}
$$

By Lemma 3.5, the permutation $\left(1_{a} \times \xi_{p-a, b} \times 1_{q-b}\right) \cdot\left(\eta_{1}^{-1} \times \eta_{2}^{-1}\right)$ is the inverse of an $(a+b, p+q-a-b)$-shuffle. Therefore, by the uniqueness in Lemma 3.2,

$$
\begin{aligned}
& \eta=\left(\eta_{1} \times \eta_{2}\right) \cdot\left(1_{a} \times \xi_{b, p-a} \times 1_{q-b}\right), \quad \alpha=\left(\xi_{1} \cdot\left(f_{(a)}^{\prime} \times g_{(b)}^{\prime}\right), \quad\right. \text { and } \\
& \beta=\xi_{2} \cdot\left(f_{(p-a)}^{\prime \prime} \times g_{(q-b)}^{\prime \prime}\right) .
\end{aligned}
$$

This proves that the summand $\alpha \otimes \beta$ appears in $\Delta(f) * \Delta(g)$. 
Consider the following graded subspace of $\mathcal{P}$ :

$$
\mathcal{S}:=\bigoplus_{n \geq 0} \mathbb{k} S_{n} .
$$

As mentioned in the introduction, the space $\mathcal{S}$ carries a Hopf algebra structure, first defined by Malvenuto and Reutenauer [21]. The following result follows by comparing (6) and (8) with the definitions in [21].

Proposition $3.7 \mathcal{S}$ is a Hopf subalgebra of $\mathcal{P}$.

Let $\sigma$ be a permutation. In the notation of [2], the element $\sigma \in \mathcal{S}$ corresponds to the basis element $F_{\sigma}^{*}$ of $\mathcal{S} S y m^{*}$, or equivalently to the element $F_{\sigma^{-1}}$ of $\mathcal{S} S y m$.

\section{Hopf algebra structure of $\mathcal{P}$}

\subsection{Inverse monoid structure and self-duality}

As $\mathcal{S}$, the Hopf algebra $\mathcal{P}$ is self-dual. To see this, recall that a block permutation is a bijection $f: \mathcal{A} \rightarrow \mathcal{B}$ between two set partitions of $[n]$. Let $\tilde{f}: \mathcal{B} \rightarrow \mathcal{A}$ denote the inverse bijection. If $f$ is uniform then so is $\tilde{f}$. The diagram of $\tilde{f} \in P_{n}$ is obtained by reflecting the diagram of $f$ across a horizontal line. Note that for $\sigma \in S_{n} \subseteq P_{n}$ we have $\tilde{\sigma}=\sigma^{-1}$.

The operation $f \mapsto \tilde{f}$ is relevant to the monoid structure of $P_{n}$. Indeed, the following properties are satisfied

$$
f=f \tilde{f} f \text { and } \tilde{f}=\tilde{f} f \tilde{f} .
$$

Together with (13) below, these properties imply that $P_{n}$ is an inverse monoid [7, Theorem 1.17]. The following properties are consequences of this fact [7, Lemma 1.18]:

$$
\widetilde{f g}=\tilde{g} \tilde{f}, \quad \tilde{\tilde{f}}=f
$$

(they can also be verified directly). It follows that $\widetilde{\sigma \cdot f}=\tilde{f} \cdot \sigma^{-1}$.

The operation $f \mapsto \tilde{f}$ is also relevant to the Hopf algebra structure of $\mathcal{P}$. Let $\mathcal{P}^{*}$ be the graded dual space of $\mathcal{P}$ :

$$
\mathcal{P}^{*}=\bigoplus_{n \geq 0}\left(\mathbb{k} P_{n}\right)^{*}
$$

Let $\left\{f^{*} \mid f \in P_{n}\right\}$ be the basis of $\left(\mathbb{k} P_{n}\right)^{*}$ dual to the basis $P_{n}$ of $\mathbb{k} P_{n}$. The product on $\mathcal{P}^{*}$ for $f^{*} \in\left(\mathbb{k} P_{n}\right)^{*}$ and $g^{*} \in\left(\mathbb{k} P_{m}\right)^{*}$ is given by

$$
f^{*} * g^{*}=\sum_{\xi \in \operatorname{Sh}(n, m)}(f \times g)^{*} \cdot \xi^{-1}
$$


Let $\Phi: \mathcal{P}^{*} \rightarrow \mathcal{P}$ be the linear map such that

$$
\Phi\left(f^{*}\right):=\tilde{f} .
$$

Proposition 4.1 The map $\Phi: \mathcal{P}^{*} \rightarrow \mathcal{P}$ is an isomorphism of graded Hopf algebras. In addition, $\Phi^{*}=\Phi$.

Proof We have

$$
\begin{aligned}
\Phi\left(f^{*} * g^{*}\right) & =\sum_{\xi \in \operatorname{Sh}(n, m)} \Phi\left((f \times g)^{*} \cdot \xi^{-1}\right) \\
& =\sum_{\xi \in S h(n, m)} \widetilde{\xi^{-1}} \cdot(\widetilde{f \times g}) \\
& =\sum_{\xi \in S h(n, m)} \xi \cdot(\tilde{f} \times \tilde{g})=\Phi(f) * \Phi(g) .
\end{aligned}
$$

Thus $\Phi$ preserves products. Since $\sim$ is an involution, $\Phi^{*}=\Phi$, and hence $\Phi$ preserves coproducts as well.

\subsection{Factorizable monoid structure and the weak order}

Let $E_{n}$ denote the poset of set partitions of $[n]$ : we say that $\mathcal{A} \leq \mathcal{B}$ if every block of $\mathcal{B}$ is contained in a block of $\mathcal{A}$. This poset is a lattice, and this structure is related to the monoid structure of uniform block permutations as follows. If $i d_{\mathcal{A}}: \mathcal{A} \rightarrow \mathcal{A}$ denotes the uniform block permutation which is the identity map on the set of blocks of $\mathcal{A}$, then

$$
i d_{\mathcal{A}} \cdot i d_{\mathcal{B}}=i d_{\mathcal{A} \wedge \mathcal{B}}
$$

In other words, viewing $E_{n}$ as a monoid under the meet operation $\wedge$, the map

$$
E_{n} \rightarrow P_{n}, \quad \mathcal{A} \mapsto i d_{\mathcal{A}},
$$

is a morphism of monoids.

Any uniform block permutation $f \in P_{n}$ decomposes (non-uniquely) as

$$
f=\sigma \cdot i d_{\mathcal{A}}
$$

for some $\sigma \in S_{n}$ and $\mathcal{A} \in E_{n}$. Note that $\sigma$ is invertible and $i d_{\mathcal{A}}$ is idempotent, by (13). It follows that $P_{n}$ is a factorizable inverse monoid [6, Section 2], [20, Chapter 2.2]. Moreover, by Lemma 2.1 in [6], any invertible element in $P_{n}$ belongs to $S_{n}$ and any idempotent element in $P_{n}$ belongs to (the image of) $E_{n}$. This lemma also guarantees that in (14), the idempotent $i d_{\mathcal{A}}$ is uniquely determined by $f$ (which is clear since $\mathcal{A}$ is the domain of $f$ ). On the other hand, $\sigma$ is not unique, and we will make a suitable choice of this factor to define a partial order on $P_{n}$. 
Consider the action of $S_{n}$ on $P_{n}$ by left multiplication. Given $\mathcal{A} \in E_{n}$, the orbit of $i d_{\mathcal{A}}$ consists of all uniform block permutations $f: \mathcal{A} \rightarrow \mathcal{B}$ with domain $\mathcal{A}$, and the stabilizer is the parabolic subgroup

$$
S_{\mathcal{A}}:=\left\{\sigma \in S_{n} \mid \sigma(A)=A \forall A \in \mathcal{A}\right\} .
$$

Consider the set of $\mathcal{A}$-shuffles:

$$
\operatorname{Sh}(\mathcal{A}):=\left\{\xi \in S_{n} \mid \text { if } i<j \text { are in the same block of } \mathcal{A} \text { then } \xi(i)<\xi(j)\right\} \text {. }
$$

It is well-known that these permutations form a set of representatives for the left cosets of the subgroup $S_{\mathcal{A}}$. Therefore, given a uniform block permutation $f: \mathcal{A} \rightarrow \mathcal{B}$ there is a unique $\mathcal{A}$-shuffle $\xi_{f}$ such that

$$
f=\xi_{f} \cdot i d_{\mathcal{A}}
$$

We use this decomposition to define a partial order on $P_{n}$ as follows:

$$
f \leq g \Longleftrightarrow f \text { and } g \text { have the same domain and } \xi_{f} \leq \xi_{g} \text {, }
$$

where the partial order on the right hand side is the left weak order on $S_{n}$ (see for instance [2]). We refer to this partial order as the weak order on $P_{n}$. Thus, $P_{n}$ is the disjoint union of certain subposets of the weak order on $S_{n}$ :

$$
P_{n} \cong \bigsqcup_{\mathcal{A} \vdash[n]} \operatorname{Sh}(\mathcal{A})
$$

(in fact, each $\operatorname{Sh}(\mathcal{A})$ is a lower order ideal in $S_{n}$ ). Figures $4-8$ show 5 of the 15 components of $P_{4}$. Note that even when $\mathcal{A}$ and $\mathcal{B}$ are set partitions of the same type the posets $\operatorname{Sh}(\mathcal{A})$ and $\operatorname{Sh}(\mathcal{B})$ need not be isomorphic.

The partial order we have defined on $P_{n}$ should not be confused with the natural partial order which is defined on any inverse semigroup [8, Chapter 7.1], [20, Chapter 1.4].

Remark 4.2 As observed by Sloane [25], there is a connection between uniform block permutations and the patience games of Aldous and Diaconis [3]. A patience game is played as follows. Start from a deck of cards numbered $1, \ldots, n$ and arranged in any order. At each step, draw a card from the top of the deck and either place it on an existing pile which shows a bigger card, or start a new pile (there are thus several choices at each step). The initial deck is a permutation of $[n]$ and the resulting piles form a set partition of $[n]$. Suppose $\xi \in S_{n}$. The set partitions $\mathcal{A}$ such that $\xi \in \operatorname{Sh}(\mathcal{A})$ are precisely the possible outputs of patience games played from a deck of cards with $\xi^{-1}(1)$ in the bottom, followed by $\xi^{-1}(2)$, up to $\xi^{-1}(n)$ on the top. Thus, uniform block permutations are in bijection with the pairs consisting of the input and the output of a patience game via $(\xi, \mathcal{A}) \leftrightarrow \xi \cdot i d_{\mathcal{A}}$. 


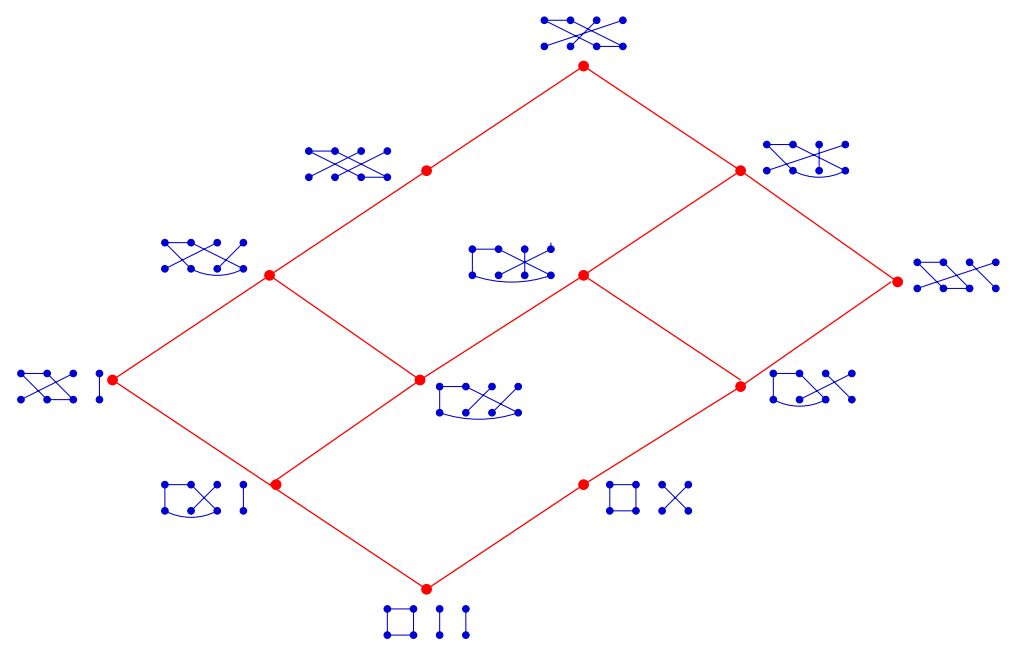

Fig. 4 The component of $P_{4}$ corresponding to $\mathcal{A}=\{1,2\}\{3\}\{4\}$

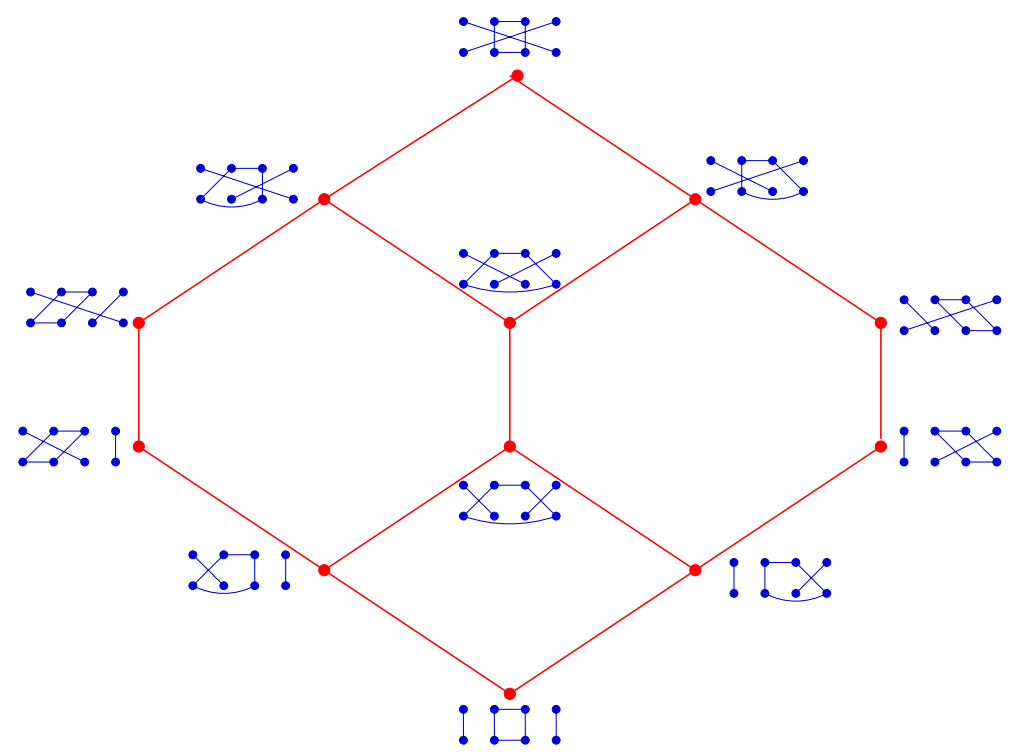

Fig. 5 The component of $P_{4}$ corresponding to $\mathcal{A}=\{1\}\{2,3\}\{4\}$

4.3 The second basis and the Hopf algebra structure

We use the weak order on $P_{n}$ to define a new linear basis of the spaces $\mathbb{k} P_{n}$, on which the algebra structure of $\mathcal{P}$ is simple. We follow the ideas of [2], where the same procedure was applied to the Hopf algebra of Malvenuto and Reutenauer. The same Möbius inversion trick has been employed frequently in inverse semigroup representation theory with a variety of purposes [19, 26-29]. 


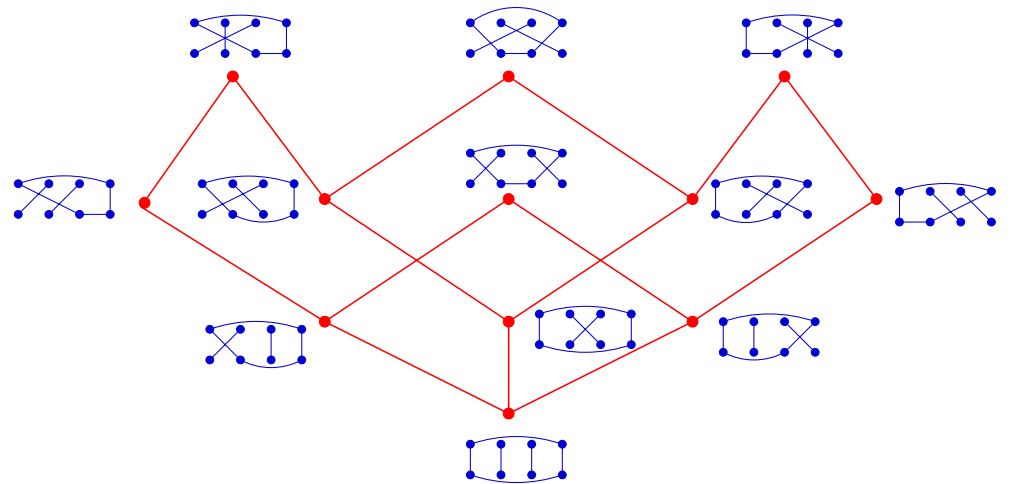

Fig. 6 The component of $P_{4}$ corresponding to $\mathcal{A}=\{1,4\}\{2\}\{3\}$

Fig. 7 The component of $P_{4}$ corresponding to $\mathcal{A}=\{1,3\}\{2\}\{4\}$

Fig. 8 The component of $P_{4}$ corresponding to $\mathcal{A}=\{1,4\}\{2,3\}$
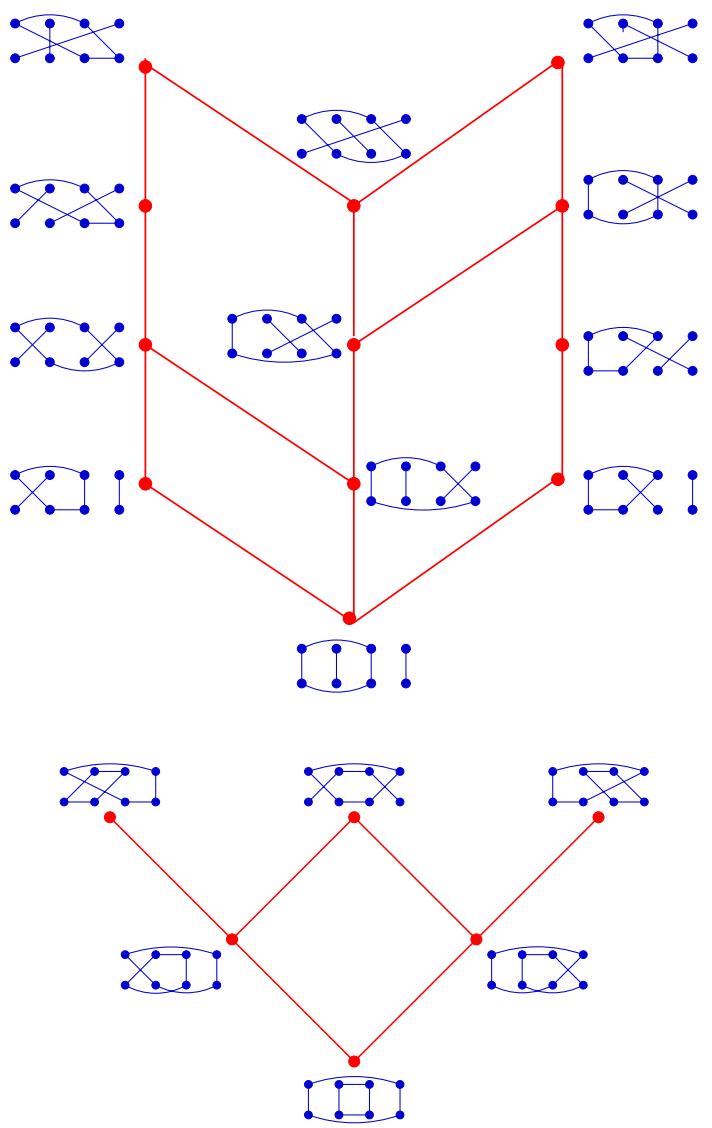

For each element $g \in P_{n}$ let

$$
X_{g}:=\sum_{f \leq g} f .
$$


By Möbius inversion, the set $\left\{X_{g} \mid g \in P_{n}\right\}$ is a linear basis of $\mathcal{P}_{n}$.

Note that all uniform block permutations $f$ in the right hand side of (17) share the same domain $\mathcal{A} \vdash[n]$, by (16).

Given set partitions $\mathcal{A} \vdash[p]$ and $\mathcal{B} \vdash[q]$, let $\mathcal{A} \times \mathcal{B}$ be the set partition of $[p+$ $q$ ] whose blocks are the blocks of $\mathcal{A}$ and the blocks $\{b+p \mid b \in B\}$ where $B$ is a block of $\mathcal{B}$. For example, if $\mathcal{A}=\{1,3,4\}\{2,5\}\{6\} \vdash[6]$ and $\mathcal{B}=\{1,4\}\{2\}\{3,5\} \vdash$ [5], then $\mathcal{A} \times \mathcal{B}=\{1,3,4\}\{2,5\}\{6\}\{7,10\}\{8\}\{9,11\} \vdash[11]$. This is compatible with concatenation of uniform block permutations in the sense that if $\mathcal{A}$ is the (co)domain of $f$ and $\mathcal{B}$ is the (co)domain of $g$, then $\mathcal{A} \times \mathcal{B}$ is the (co)domain of $f \times g$. In particular,

$$
i d_{\mathcal{A}} \times i d_{\mathcal{B}}=i d_{\mathcal{A} \times \mathcal{B}} .
$$

Recall the maximum $(p, q)$-shuffle $\xi_{p, q}$ from (9).

Lemma 4.3 Let $\lambda: \operatorname{Sh}(p, q) \times \operatorname{Sh}(\mathcal{A}) \times \operatorname{Sh}(\mathcal{B}) \longrightarrow \operatorname{Sh}(\mathcal{A} \times \mathcal{B})$ be defined by

$$
\lambda(\xi, \sigma, \tau):=\xi \cdot(\sigma \times \tau) .
$$

Endow each set of shuffles with the weak order. Then

(i) $\lambda$ is bijective;

(ii) $\lambda^{-1}$ is order preserving, that is,

$$
\xi \cdot(\sigma \times \tau) \leq \xi^{\prime} \cdot\left(\sigma^{\prime} \times \tau^{\prime}\right) \Longrightarrow \xi \leq \xi^{\prime}, \quad \sigma \leq \sigma^{\prime}, \text { and } \tau \leq \tau^{\prime} ;
$$

(iii) $\lambda$ is order preserving when restricted to any of the following sets:

$$
\left\{\xi_{p, q}\right\} \times \operatorname{Sh}(\mathcal{A}) \times \operatorname{Sh}(\mathcal{B}), \quad\left\{1_{p+q}\right\} \times \operatorname{Sh}(\mathcal{A}) \times \operatorname{Sh}(\mathcal{B}), \text { or } \operatorname{Sh}(p, q) \times\{(\sigma, \tau)\},
$$

for any $\sigma \in \operatorname{Sh}(\mathcal{A}), \tau \in \operatorname{Sh}(\mathcal{B})$.

Proof The proof is similar to that of Proposition 2.5 in [2].

The product of $\mathcal{P}$ takes the following simple form on the $X$-basis.

Proposition 4.4 Let $g_{1} \in P_{p_{1}}$ and $g_{2} \in P_{p_{2}}$ be uniform block permutations. Then

$$
X_{g_{1}} * X_{g_{2}}=X_{\xi_{p, q} \cdot\left(g_{1} \times g_{2}\right)} \text {. }
$$

Proof We have:

$$
X_{g_{1}} * X_{g_{2}} \stackrel{(17)}{=} \sum_{\substack{f_{1} \leq g_{1} \\ f_{2} \leq g_{2}}} f_{1} * f_{2} \stackrel{(6)}{=} \sum_{\xi \in \operatorname{Sh}\left(p_{1}, p_{2}\right)} \sum_{\substack{f_{1} \leq g_{1} \\ f_{2} \leq g_{2}}} \xi \cdot\left(f_{1} \times f_{2}\right) \text {. }
$$

Let $\mathcal{A}_{i}$ be the domain of $g_{i}, i=1,2$. Choose $\xi \in \operatorname{Sh}\left(p_{1}, p_{2}\right)$ and $f_{i} \leq g_{i}$ in $P_{p_{i}}$, $i=1,2$. Then $\mathcal{A}_{i}$ is the domain of $f_{i}$, and by (15),

$$
f_{i}=\xi_{f_{i}} \cdot i d_{\mathcal{A}_{i}} .
$$


Hence

$$
\xi \cdot\left(f_{1} \times f_{2}\right)=\xi \cdot\left(\xi_{f_{1}} \times \xi_{f_{2}}\right) \cdot\left(i d_{\mathcal{A}_{1}} \times i d_{\mathcal{A}_{2}}\right) .
$$

Since $f_{i} \leq g_{i}$, we have $\xi_{f_{i}} \leq \xi_{g_{i}}$, by (16). Therefore, by Lemma 4.3.(iii),

$$
\xi \cdot\left(\xi_{f_{1}} \times \xi_{f_{2}}\right) \leq \xi_{p, q} \cdot\left(\xi_{g_{1}} \times \xi_{g_{2}}\right) .
$$

Hence, by (16),

$$
\xi \cdot\left(f_{1} \times f_{2}\right) \leq \xi_{p, q} \cdot\left(g_{1} \times g_{2}\right) .
$$

Thus every summand in $X_{g_{1}} * X_{g_{2}}$ occurs in $X_{\xi_{p, q} \cdot\left(g_{1} \times g_{2}\right)}$.

Conversely, let $f$ be a summand in $X_{\xi_{p, q} \cdot\left(g_{1} \times g_{2}\right)}$. Since the domain of $\xi_{p, q} \cdot\left(g_{1} \times\right.$ $\left.g_{2}\right)$ is $\mathcal{A}_{1} \times \mathcal{A}_{2}$, we have $f=\xi_{f} \cdot i d_{\mathcal{A}_{1} \times \mathcal{A}_{2}}$. Hence

$$
\xi_{f} \leq \xi_{p, q} \cdot\left(\xi_{g_{1}} \times \xi_{g_{2}}\right)
$$

and from (i) and (ii) in Lemma 4.3 we obtain $\xi \in \operatorname{Sh}\left(p_{1}, p_{2}\right), \sigma_{i} \in \operatorname{Sh}\left(\mathcal{A}_{i}\right)$ such that

$$
\sigma_{i} \leq \xi_{g_{i}} \quad \text { and } \quad \xi_{f}=\xi \cdot\left(\sigma_{1} \times \sigma_{2}\right)
$$

Let $f_{i}:=\sigma_{i} \cdot i d_{\mathcal{A}_{i}}$. Then

$$
f_{i} \leq g_{i} \quad \text { and } \quad f=\xi \cdot\left(f_{1} \times f_{2}\right),
$$

which shows that every summand in $X_{\xi_{p, q} \cdot\left(g_{1} \times g_{2}\right)}$ occurs in $X_{g_{1}} * X_{g_{2}}$.

From Propositions 4.4 and 4.1 we deduce:

Corollary 4.5 The Hopf algebra $\mathcal{P}$ is free as an algebra and cofree as a graded coalgebra.

Let $V$ denote the space of primitive elements of $\mathcal{P}$. It follows that the generating series of $\mathcal{P}$ and $V$ are related by

$$
\mathcal{P}(x)=\frac{1}{1-V(x)} .
$$

Since

$$
\mathcal{P}(x)=1+x+3 x^{2}+16 x^{3}+131 x^{4}+1496 x^{5}+22482 x^{6}+\cdots
$$

we deduce that

$$
V(x)=x+2 x^{2}+11 x^{3}+98 x^{4}+1202 x^{5}+19052 x^{6}+\cdots .
$$

Let $X_{f}^{*}$ be the linear basis of $\mathcal{P}^{*}$ dual to the basis $X_{f}$ of $\mathcal{P}$ and let $M_{f}$ be the image of $X_{f}^{*}$ under the isomorphism $\Phi: \mathcal{P}^{*} \rightarrow \mathcal{P}$ of Proposition 4.1. Explicitly, the basis $M_{f}$ is uniquely determined by the equations

$$
\tilde{f}=\sum_{f \leq g} M_{g}
$$


that hold for every $f \in \mathcal{P}$. It follows that the elements $M_{f}$, as $f$ runs over all uniform block permutations that cannot be decomposed as

$$
f=\xi_{p, q} \cdot\left(f_{1} \times f_{2}\right)
$$

with $p$ and $q>0$, form linear basis of the space of primitive elements of $\mathcal{P}$. A uniform block permutation $f \in P_{n}$ can be decomposed in this manner if and only if there is $0<p<n$ such that the first $p$ elements of the domain of $f$ are not connected to any of the first $n-p$ elements of the codomain. A permutation $\sigma \in S_{n}$ satisfies this condition if and only if $\sigma$ has a global descent at $p$, in agreement with [2, Corollary 6.3].

Remark 4.6 A similar conclusion may be derived by introducing another basis

$$
Z_{g}:=\sum_{g \leq f} f .
$$

This has the property that

$$
Z_{g_{1}} * Z_{g_{2}}=Z_{g_{1} \times g_{2}} .
$$

Note that $Z_{i d_{\mathcal{A}}}$ is the element $Z_{\mathcal{A}}$ introduced in (4) and so denoted throughout Section 2.3.

\section{The Hopf algebra of symmetric functions in non-commuting variables}

Let $X$ be a countable set, the alphabet. A word of length $n$ is a function $w:[n] \rightarrow X$. Let $\mathbb{k}\langle\langle X\rangle\rangle$ be the algebra of non-commutative power series on the set of variables $X$. Its elements are infinite linear combinations of words, finitely many of each length, and the product is concatenation of words.

The kernel of a word $w$ of length $n$ is the set partition $\mathcal{K}(w)$ of $[n]$ whose blocks are the non-empty fibers of $w$. Order the set of set partitions of $[n]$ by refinement, as in Section 4.2. For each set partition $\mathcal{A}$ of $[n]$, let

$$
p_{A}:=\sum_{\mathcal{K}(w) \leq A} w \in \mathbb{k}\langle\langle X\rangle\rangle .
$$

This is the sum of all words $w$ such that if $i$ and $j$ are in the same block of $\mathcal{A}$ then $w(i)=w(j)$. For instance

$$
p_{\{1,3\}\{2,4\}}=x y x y+x z x z+y x y x+\cdots+x^{4}+y^{4}+z^{4}+\cdots .
$$

The subspace of $\mathbb{k}\langle\langle X\rangle\rangle$ linearly spanned by the elements $p_{\mathcal{A}}$ as $\mathcal{A}$ runs over all set partitions of $[n], n \geq 0$, is a subalgebra $\Pi$ of $\mathbb{k}\langle\langle X\rangle\rangle$, graded by length. The elements of $\Pi$ can be characterized as those power series of finite degree that are invariant under any permutation of the variables. $\Pi$ is the algebra of symmetric functions in non-commuting variables introduced by Wolf [31] and recently studied by Gebhard, 
Rosas, and Sagan $[11,12,23]$ in connection to Stanley's chromatic symmetric function.

The algebra $\Pi$ is in fact a graded Hopf algebra $[1,4,5]$. The coproduct is defined via evaluation of symmetric functions on two copies of the alphabet $X$. In order to describe the product and coproduct of $\Pi$ on the basis elements $p_{\mathcal{A}}$ we introduce some notation.

To a set partition $\mathcal{A} \vdash[n]$ and a subset of blocks $S \subseteq \mathcal{A}$ we associate a new set partition $\mathcal{A}_{S}$ as follows. Write

$$
\bigcup_{A \in S} A=\left\{j_{1}, \cdots, j_{S}\right\} \subseteq[n]
$$

with $j_{1}<\cdots<j_{s}$. The set $S$ is a partition of $\left\{j_{1}, \cdots, j_{s}\right\}$. We turn it into a partition $\mathcal{A}_{S}$ of $[s]$ by replacing each $j_{i}$ by $i$ for $1 \leq i \leq m$ and preserving the block structure. For instance, if $\mathcal{A}=\{1,5\}\{2,4,6\}\{3,7\}$ and $S=\{1,5\}\{3,7\}$, then $\mathcal{A}_{S}=\{1,3\}\{2,4\} \vdash[4]$.

The product and coproduct of $\Pi$ are given by

$$
\begin{aligned}
p_{\mathcal{A}} p_{\mathcal{B}} & =p_{\mathcal{A} \times \mathcal{B}}, \\
\Delta\left(p_{\mathcal{A}}\right) & =\sum_{S \sqcup T=\mathcal{A}} p_{\mathcal{A}_{S}} \otimes p_{\mathcal{A}_{T}},
\end{aligned}
$$

the sum over all decompositions of $\mathcal{A}$ into disjoint sets of blocks $S$ and $T$. For example, if $\mathcal{A}=\{1,2,6\}\{3,5\}\{4\}$, then

$$
\begin{aligned}
\Delta\left(p_{\mathcal{A}}\right)= & p_{\mathcal{A}} \otimes 1+p_{\{1,2,5\}\{3,4\}} \otimes p_{\{1\}}+p_{\{1,2,4\}\{3\}} \otimes p_{\{1,2\}}+p_{\{1,3\}\{2\}} \otimes p_{\{1,2,3\}} \\
& +p_{\{1,2,3\}} \otimes p_{\{1,3\}\{2\}}+p_{\{1,2\}} \otimes p_{\{1,2,4\}\{3\}} \\
& +p_{\{1\}} \otimes p_{\{1,2,5\}\{3,4\}}+1 \otimes p_{\mathcal{A}} .
\end{aligned}
$$

Consider now the direct sum of the subspaces $\mathcal{Z}_{n}$ of $\mathbb{k} P_{n}$ introduced in Section 2.3:

$$
\mathcal{Z}:=\bigoplus_{n \geq 0} \mathcal{Z}_{n} \subset \mathcal{P}
$$

The elements $Z_{\mathcal{A}}$ defined in (4) form a linear basis of $\mathcal{Z}_{n}$.

Theorem $5.1 \mathcal{Z}$ is a Hopf subalgebra of $\mathcal{P}$. Moreover, the map

$$
\Theta: \mathcal{Z} \rightarrow \Pi, \quad \Theta\left(Z_{\mathcal{A}}\right):=p_{\mathcal{A}}
$$

is an isomorphism of graded Hopf algebras.

Proof Recall from Remark 4.6 that $Z_{\mathcal{A}}=Z_{i d_{\mathcal{A}}}$, and therefore

$$
Z_{\mathcal{A}} * Z_{\mathcal{B}}=Z_{\mathcal{A} \times \mathcal{B}}
$$

Thus $\Theta$ is a morphism of algebras. 
To prove that $\Theta$ is a morphism of coalgebras we need to show that

$$
\Delta\left(Z_{\mathcal{A}}\right)=\sum_{S \sqcup T=\mathcal{A}} Z_{\mathcal{A}_{S}} \otimes Z_{\mathcal{A}_{T}}
$$

for every set partition $\mathcal{A} \vdash[n]$. We have

$$
Z_{\mathcal{A}}=\sum_{i d_{\mathcal{A}} \leq f} f=\sum_{\xi \in \operatorname{Sh}(\mathcal{A})} \xi \cdot i d_{\mathcal{A}}
$$

and hence

$$
Z_{\mathcal{A}_{S}}=\sum_{\sigma \in \operatorname{Sh}\left(\mathcal{A}_{S}\right)} \sigma \cdot i d_{\mathcal{A}_{S}} \quad \text { and } \quad Z_{\mathcal{A}_{T}}=\sum_{\tau \in \operatorname{Sh}\left(\mathcal{A}_{T}\right)} \tau \cdot i d_{\mathcal{A}_{T}} .
$$

Fix a decomposition $\mathcal{A}=S \sqcup T$ and consider the summand in $Z_{\mathcal{A}_{S}} \otimes Z_{\mathcal{A}_{T}}$ indexed by shuffles $\sigma \in \operatorname{Sh}\left(\mathcal{A}_{S}\right)$ and $\tau \in \operatorname{Sh}\left(\mathcal{A}_{T}\right)$. Out of this data we construct shuffles $\eta$ and $\xi$ as follows. First we write

$$
\bigcup_{A \in S} A=\left\{j_{1}, \cdots, j_{S}\right\} \quad \text { and } \bigcup_{A \in T} A=\left\{k_{1}, \cdots, k_{t}\right\}
$$

with $j_{1}<\cdots<j_{s}$ and $k_{1}<\cdots<k_{t}$. We let $\eta$ be the unique $(s, t)$-shuffle such that $\eta([s])=\left\{j_{1}, \cdots, j_{s}\right\}$ (and hence $\left.\eta([s+1, n])=\left\{k_{1}, \cdots, k_{t}\right\}\right)$, and we define $\xi$ by

$$
\xi\left(j_{i}\right)=\sigma(i) \forall i \in[s] \quad \text { and } \quad \xi\left(k_{i}\right)=s+\tau(i) \forall i \in[t]
$$

Then $\xi$ is increasing in each block of $\mathcal{A}$, so $\xi \in \operatorname{Sh}(\mathcal{A})$, and by construction

$$
\xi \cdot i d_{\mathcal{A}} \cdot \eta=\left(\sigma \cdot i d_{\mathcal{A}_{S}}\right) \times\left(\tau \cdot i d_{\mathcal{A}_{T}}\right) .
$$

Therefore, by Lemma 3.2, $s$ is a breaking point of $\xi \cdot i d_{\mathcal{A}}$, and by (8), $\left(\sigma \cdot i d_{\mathcal{A}_{S}}\right) \otimes$ $\left(\tau \cdot i d_{\mathcal{A}_{T}}\right)$ is a summand in $\Delta\left(Z_{\mathcal{A}}\right)$.

A similar argument shows that, conversely, every summand in $\Delta\left(Z_{\mathcal{A}}\right)$ occurs in this manner.

Thus the Hopf algebra of uniform block permutations $\mathcal{P}$ contains the Hopf algebra $\Pi$ of symmetric functions in non-commuting variables. Note also that this reveals the existence of a second operation on $\Pi$ : according to Corollary 2.2, each homogeneous component $\Pi_{n}$ carries an associative non-unital product that turns it into a right ideal of the monoid algebra $\mathbb{k} P_{n}$.

\section{References}

1. Aguiar, M., Mahajan, S.: Coxeter Groups and Hopf Algebras. Fields Institute Monographs, vol. 23. AMS, Providence (2006)

2. Aguiar, M., Sottile, F.: Structure of the Malvenuto-Reutenauer Hopf algebra of permutations. Adv. Math. 191(2), 225-275 (2005) 
3. Aldous, D., Diaconis, P.: Longest increasing subsequences: from patience sorting to the Baik-DeiftJohansson theorem. Bull. Am. Math. Soc. 36, 413-432 (1999)

4. Bergeron, N., Hohlweg, C., Rosas, M., Zabrocki, M.: Grothendieck bialgebras, partition lattices and symmetric functions in noncommutative variables. Electron. J. Comb. 13(1), 75 (2006) (electronic)

5. Bergeron, N., Zabrocki, M.: The Hopf algebras of symmetric functions and quasisymmetric functions in non-commutative variables are free and cofree. math.CO/0509265 (2005)

6. Chen, S.Y., Hsieh, S.C.: Factorizable inverse semigroups. Semigroup Forum 8(4), 283-297 (1974)

7. Clifford, A.H., Preston, G.B.: The Algebraic Theory of Semigroups, vol. I. Mathematical Surveys, vol. 7. American Mathematical Society, Providence (1961)

8. Clifford, A.H., Preston, G.B.: The Algebraic Theory of Semigroups, vol. II. Mathematical Surveys, vol. 7. American Mathematical Society, Providence (1967)

9. Easdown, D., East, J., FitzGerlad, D.G.: Presentations of factorizable inverse monoids (2004)

10. FitzGerald, D.G.: A presentation for the monoid of uniform block permutations. Bull. Austral. Math. Soc. 68, 317-324 (2003)

11. Gebhard, D.D., Sagan, B.E.: Sinks in acyclic orientations of graphs. J. Comb. Theory B 80, 130-146 (2000)

12. Gebhard, D.D., Sagan, B.E.: A chromatic symmetric function in noncommuting variables. J. Algebr. Comb. 13, 227-255 (2001)

13. Grood, C.: The rook partition algebra. J. Comb. Theory Ser. A 113(2), 325-351 (2006)

14. Halverson, T.: Representations of the $q$-rook monoid. J. Algebra 273(1), 227-251 (2004)

15. Halverson, T., Ram, A.: $q$-rook monoid algebras. Hecke algebras, and Schur-Weyl duality. Zap. Nauchn. Sem. S.-Peterburg. Otdel. Mat. Inst. Steklov. (POMI) 283 (2001), Teor. Predst. Din. Sist. Komb. Algoritm. Metody. 6, 224-250, 262-263; translation in J. Math. Sci. (N.Y.) 121(3), 24192436 (2004)

16. Jones, V.F.R.: The Potts model and the symmetric group. In: Subfactors: Proceedings of the Tanaguchi Symposium on Operator Algebras, Kyuzeso, 1993, pp. 259-267. World Scientific, River Edge (1994)

17. Kosuda, M.: Characterization for the party algebra. Ryukyu Math. J. 13, 7-22 (2000)

18. Kosuda, M.: Party algebra and construction of its irreducible representations. Paper presented at Formal Power Series and Algebraic Combinatorics (FPSAC01), Tempe, Arizona (USA), 20-26 May 2001

19. Kudryavtseva, G., Mazorchuk, V.: Schur-Weyl dualities for symmetric inverse semigroups, math.RT/0702864

20. Lawson, M.V.: Inverse Semigroups. The Theory of Partial Symmetries. World Scientific, River Edge (1998)

21. Malvenuto, C., Reutenauer, C.: Duality between quasi-symmetric functions and the Solomon descent algebra. J. Algebra 177(3), 967-982 (1995)

22. Martin, P.P.: Temperley-Lieb algebras for non-planar statistical mechanics - the partition algebra construction. J. Knot Theory Appl. 3(1), 51-82 (1994)

23. Rosas, M., Sagan, B.: Symmetric functions in non-commuting variables. Trans. Am. Math. Soc. 358(1), 215-232 (2006)

24. Sixdeniers, J.-M., Penson, K.A., Solomon, A.I.: Extended Bell and Stirling numbers from hypergeometric exponentiation. J. Integer Seq. 4, 01.1 .4 (2001)

25. Sloane, N.J.A.: An on-line version of the encyclopedia of integer sequences. Electron. J. Comb. 1 (1994), Feature 1, approx. 5 pp. (electronic), http://akpublic.research.att.com/ njas/sequences/ol.html

26. Solomon, L.: The Burnside algebra of a finite group. J. Comb. Theory 2, 603-615 (1967)

27. Solomon, L.: Representations of the rook monoid. J. Algebra 256(2), 309-342 (2002)

28. Steinberg, B.: Möbius functions and semigroup representation theory. J. Comb. Theory Ser. A 113(5), 866-881 (2006)

29. Steinberg, B.: Möbius functions and semigroup representation theory, II: character formulas and multiplicities. math.CO/0607564

30. Tanabe, K.: On the centralizer algebra of the unitary reflection group $G(m, p, n)$. Nagoya Math. J. 148, 113-126 (1997)

31. Wolf, M.C.: Symmetric functions of non-commuting elements. Duke Math. J. 2, 626-637 (1936) 